\title{
Iterative BCJR Equalizer for Underwater Communication
}

\author{
Tae-Seok Ahn ${ }^{1, \text { a }}$, Ha-Hyun Sung ${ }^{2, b}$, and Ji-Won Jung ${ }^{3, c}$
}

${ }^{123}$ Department of Radio Communication Engineering, Korea Maritime and Ocean University, Busan 606-791, Korea

a ats320@kmou.ac.kr, ${ }^{b}$ hhsung@kmou.ac.kr, ${ }^{c}$ jwjung@kmou.ac.kr

Corresponding Author: Ji-Won Jung

\begin{abstract}
Keywords: Convolutional codes, BCJR decoder, BCJR Equalization, Decision Feedback Equalizer,
\end{abstract} Underwater communication.

\begin{abstract}
In this paper, we presented an iterative BCJR equalization to cope with inter symbol interference induced by reflection of sea-level and sea-bottom for underwater communication channel. Iterative BCJR equalizer consists of inner codes and outer codes, we employ decision feedback equalizer as an outer codes and half rate convolutional codes as inner codes. Equalizer and BCJR decoder are connected through the interleaving and de-interleaving that update each other's information repeatedly. At the receiver side, we resort to powerful BCJR equalization algorithms that iteratively exchange probabilistic information between inner decoder and outer decoder, thereby the error rates can be reduced significantly. Based on experimental channel response, we confirmed that the performance is improved as iteration number is increased.
\end{abstract}

\section{Introduction}

It is well known that underwater (UW) channels are often hostile for UW sensor communications, which impose three major obstacles for coherent transceivers. One is the excessive multipath delay spread in UW channel which usually causes the inter-symbol interference (ISI). Another obstacle is the Doppler shift due to source-receiver relative motion, which causes compression or dilation on the received signals. The last one is the fast time-varying phase drift due to random medium of the UW acoustic channels. Various methods to cope with multipath effect have been developed. In addition to ISI, co-channel interference (CoI) is also occurred results from the use of multiple transmitters in UW sensor communication. Removal of both $\mathrm{CoI}$ and ISI is a challenging problem in view of poor channel conditions. The optimal detector is a maximum likelihood detector (MLD), which can be realized for example by a soft Viterbi algorithm. Due to the length of the impulse response in the UW channel, the number of states in the decoder will be increased. A well-known method to counteract ISI is the decision feedback equalizer (DFE), which has been used in many UW sensor communication links [1,2]. However the use of DFE has difficulties when multipath with a number of arrivals has equal strength or low SNR. The other way to cope with ISI is iterative equalizer which consists of an outer loop in the receiver. An inner loop consists of BCJR decoder. The assembly utilizes error correcting capability of the convolutional codes to get an efficient equalizer [3,4]. In this paper, we employ the iterative receiver structure and present more details on the structure and algorithms. This structure with fine-tuned parameters is employed to process experimental data of fixed source to fixed receiver at transmission data rate of $1 \mathrm{k} \mathrm{symbol} / \mathrm{s}$. The results indicate that the scheme effectively works with coded bit error rate (BER) on the order of zero. It shows that how much coding gains can be obtained as iteration number is increased.

\section{System Model}

The baseband model of BCJR equalizer is shown in Fig. 1. Fig. 1 shows iterative linear equalizer that is decision feedback equalizer, which constitutes an outer code of the receiver. An inner code consists of convolutional codes. The information data to be transmitted are encoded by half rate convolutional codes with constraint length of 7 . 


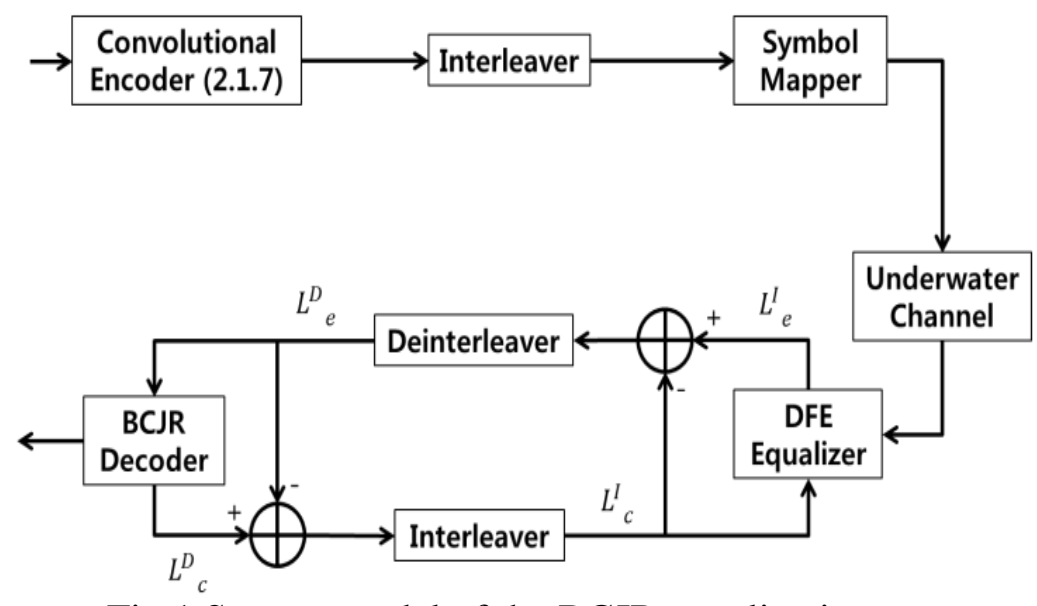

Fig.1 System model of the BCJR equalization

Convolutional codes. Convolutional codes are used extensively in numerous applications in order to achieve reliable data transmission, including wireless and underwater communication. Especially, a popular convolutional code used a constraint length $\mathrm{k}$ of 7 and rate of $1 / 2$. This paper used (2.1.7) convolutional code with generator matrix of $(171,133)_{\text {octal }}$ [5]

Interleaver. The interleavers are designed for good properties in convolutional codes. The receiver of BCJR equalizer consists of equalizer and decoder. Equalizer and BCJR decoder are connected through the interleaving and de-interleaving that update each other's information repeatedly. The inner coded bits are then subtracted from the input and interleaved. The interleaved output is canceled a posteriori from the proceeding received signal. Interleaving helps receiver convergence.

Decision Feedback Equalizer. The $\mathrm{L}_{\mathrm{e}}^{\mathrm{I}}$ is output value of DFE as estimated extrinsic value form received signal. Let $\mathrm{y}[\mathrm{k}]$ be the equalizer input at time $\mathrm{k}$, and then the output of the DFE at time $\mathrm{k}$. $\mathrm{L}_{\mathrm{e}}^{\mathrm{I}}[\mathrm{k}]$ is given by

$$
L_{e}^{I}[k]=\sum_{i=0}^{N_{b}-1} c_{i}[k] y[k-i]-\sum_{j=0}^{N_{a}} b_{j}[k] \widehat{L}_{e}^{I}[k-j] .
$$

Where, $c_{i}[\mathrm{k}]\left(\mathrm{i}=0,1, \cdots, \mathrm{N}_{\mathrm{c}-1}\right)$ are the forward equalizer taps at time $\mathrm{k}, \mathrm{b}_{\mathrm{j}}[\mathrm{k}]\left(\mathrm{j}=0,1, \cdots, \mathrm{N}_{\mathrm{b}}\right)$ are the feedback taps at time $\mathrm{k}$, and $\hat{\mathrm{L}}_{\mathrm{e}}^{\mathrm{I}}[\mathrm{k}]$ is the slicer output, which is the constellation point closest to $\mathrm{L}_{\mathrm{e}}^{\mathrm{I}}[\mathrm{k}]$. $\mathrm{e}_{\mathrm{D}}[\mathrm{k}]=\mathrm{L}_{\mathrm{e}}^{\mathrm{I}}[\mathrm{k}]-\hat{\mathrm{L}}_{\mathrm{e}}^{\mathrm{I}}[\mathrm{k}]$ is the decision error[6][7]. The value of $\mathrm{L}_{\mathrm{e}}^{\mathrm{D}}$ after de-interleaving is computed, $\mathrm{L}_{\mathrm{e}}^{\mathrm{I}}-\mathrm{L}_{\mathrm{c}}^{\mathrm{I}}$, then input BCJR decoder. The estimated extrinsic value of $\mathrm{L}_{\mathrm{c}}^{\mathrm{D}}$ at decoder output is given by

$$
L_{c}^{D}=\log \frac{\mathrm{P}(\mathrm{x}=+1)}{\mathrm{P}(\mathrm{x}=-1)}
$$

The extrinsic value $\mathrm{L}_{\mathrm{c}}^{\mathrm{D}}$ of which calculates the post probability is error correction terms. The re-interleaving and of computed value as $\mathrm{L}_{c}^{\mathrm{D}}-\mathrm{L}_{\mathrm{e}}^{\mathrm{D}}$ is input to DFE, then $\mathrm{L}_{\mathrm{c}}^{\mathrm{I}}$ is updated in order to compensate for the errors.

BCJR Decoder. Generally, the decoding method of the convolutional code is a Viterbi decoding using a hard decision value. However, to improve performance through iteration with DFE, soft decision value is required. Therefore, we used BCJR algorithm with soft decision value. BCJR algorithm is a well-known maximum a posteriori probability decoding algorithm which has been proposed earlier for point to point communication applications $[8,9,10]$.

A set of triples (previous state, channel output, next state) uniquely defines a finite state machine on which the BCJR operates. As an illustration, a trellis is shown in Fig.2. It has 64 states $\left(s_{0}, s_{1}, \ldots, s_{63}\right)$, and each state is given by a different 6-bit pattern. The states in vertical columns represent all possible states that the channel (or FSM) can take at a given time instant, while the labeled edges represent possible transitions. Thus, neighboring columns represent consecutive time 
instants. Given $\mathrm{s}^{\prime}$-the previous state, $\mathrm{s}=\left(\mathrm{u}_{\mathrm{j}-\mathrm{m}}, \mathrm{u}_{\mathrm{j}-\mathrm{m}+1}, \ldots, \mathrm{u}_{\mathrm{j}}, \mathrm{u}_{\mathrm{j}+1}, \ldots, \mathrm{u}_{\mathrm{j}+\mathrm{m}}\right)$-the present state, $\mathrm{u}=\left(\mathrm{u}_{1}, \mathrm{u}_{2}, \ldots, \mathrm{u}_{\mathrm{n}}\right)$-the transmitted code-word, and $\mathrm{y}=\left(\mathrm{y}_{1}, \mathrm{y}_{2}, \ldots, \mathrm{y}_{\mathrm{n}}\right)$-the received sequence, the log-likelihood ratio (LLR) (denoting the bit reliability) of $u_{j}(j=1,2, \ldots, n)$, is calculated as

$$
\begin{aligned}
& \mathrm{L}\left(\mathrm{u}_{\mathrm{j}}\right)=\max ^{*}\left[\alpha_{\mathrm{j}-1}\left(\mathrm{~s}^{\prime}\right)+\gamma_{\mathrm{j}}\left(\mathrm{s}^{\prime}, \mathrm{s}\right)+\beta_{\mathrm{j}}(\mathrm{s})\right]\left(\mathrm{s}^{\prime}, \mathrm{s}\right): \mathrm{u}_{\mathrm{j}}=0 \\
& -\max ^{*}\left[\alpha_{\mathrm{j}-1}\left(\mathrm{~s}^{\prime}\right)+\gamma_{\mathrm{j}}\left(\mathrm{s}^{\prime}, \mathrm{s}\right)+\beta_{\mathrm{j}}(\mathrm{s})\right]\left(s^{\prime}, \mathrm{s}\right): \mathrm{u}_{\mathrm{j}}=1
\end{aligned}
$$

The dashed line corresponds to transitions $\left(\mathrm{s}^{\prime}, \mathrm{s}\right): \mathrm{u}_{\mathrm{j}}=0$, and the solid lines correspond to transitions $\left(\mathrm{s}^{\prime}, \mathrm{s}\right): \mathrm{u}_{\mathrm{j}}=1$ in Fig. 2. The forward metric $\alpha_{j}(s)$, backward metric $\beta_{j-1}\left(s^{\prime}\right)$, and branch metric $\gamma_{\mathrm{j}}\left(\mathrm{s}^{\prime}, \mathrm{s}\right)$ are given by

$$
\begin{aligned}
& \alpha_{j}(s)=\max ^{*}\left[\alpha_{j-1}\left(s^{\prime}\right)+\gamma_{j}\left(s^{\prime}, s\right)\right] \\
& \beta_{j-1}\left(s^{\prime}\right)=\max ^{*}\left[\beta_{j}(s)+\gamma_{j}\left(s^{\prime}, s\right)\right] \\
& \gamma_{j}\left(s^{\prime}, s\right)=\log p\left(s_{j}=s, y_{j} \mid s_{j-1}=s^{\prime}\right)=\log p\left(y_{j} \mid u_{j}\right) p\left(u_{j}\right)
\end{aligned}
$$

The $\max ^{*}$-operator is defined as

$$
\max ^{*}(x, y)=\max (x, y)+\log \left(1+e^{|x-y|}\right)
$$

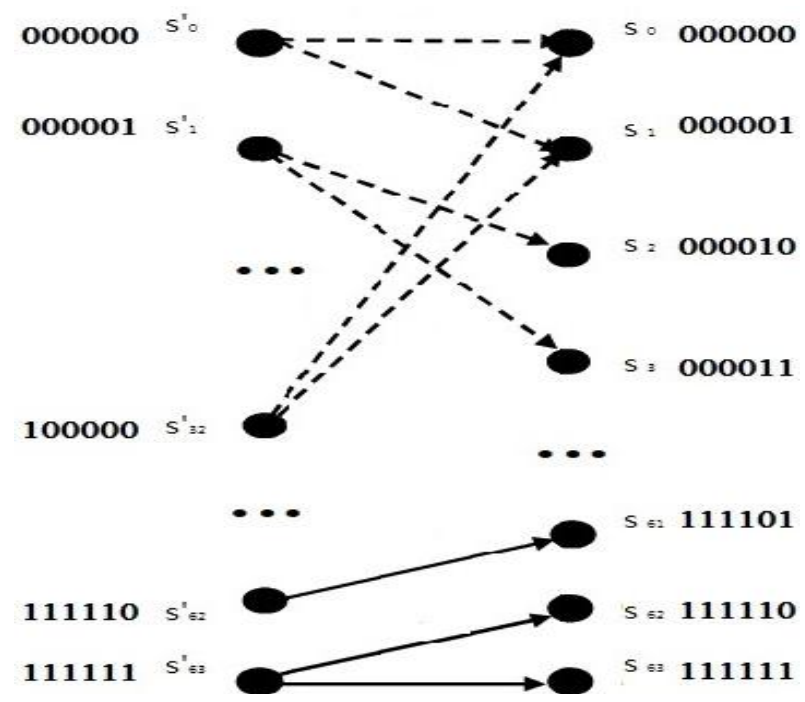

Fig.2 Trellis structure of BCJR (2.1.7)

\section{Experimental Results}

We evaluate the performance of the proposed method in real underwater environments. The experiment was conducted on a lake of Munkyeong city, Korea, in March 2014. The water depth was approximately 40 [m]. Fig. 3 shows depiction of lake trial. 


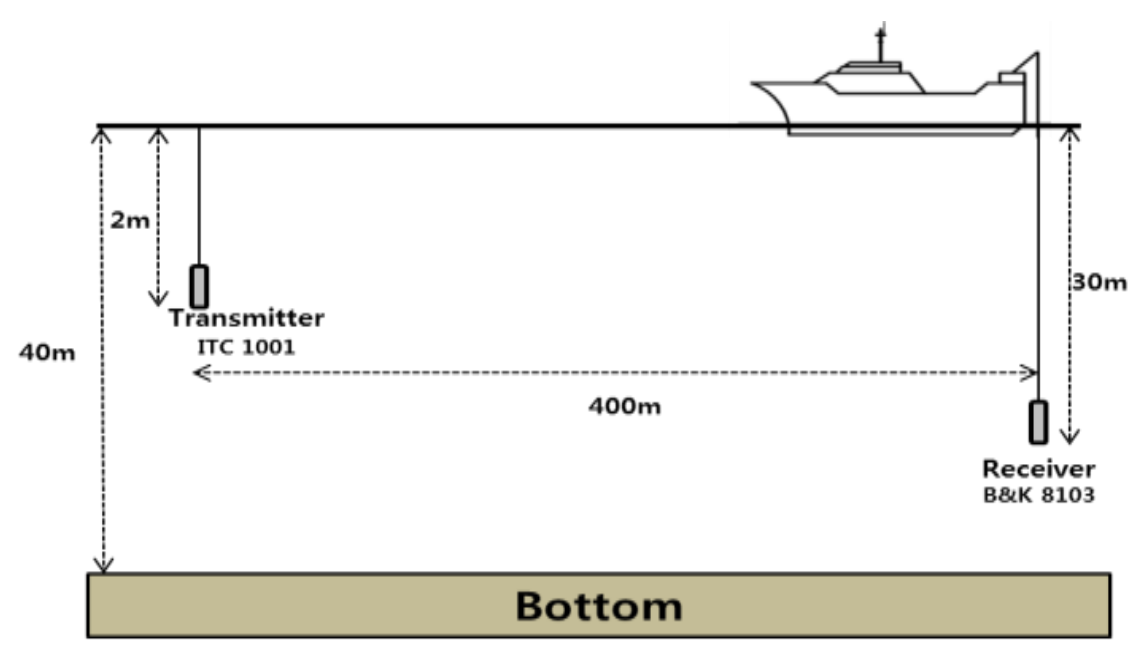

Fig.3 Depiction of lake trial

The source signal has $16[\mathrm{kHz}]$ center frequency and 1 [kbps] data rate. The hydrophone was equipped at $30[\mathrm{~m}]$ lake bottom and the horizontal range from transducer was $400[\mathrm{~m}]$. The received signal was sampled at $192[\mathrm{kHz}]$ sampling frequency. The packet structure of transmitted signals is shown in Fig. 4.

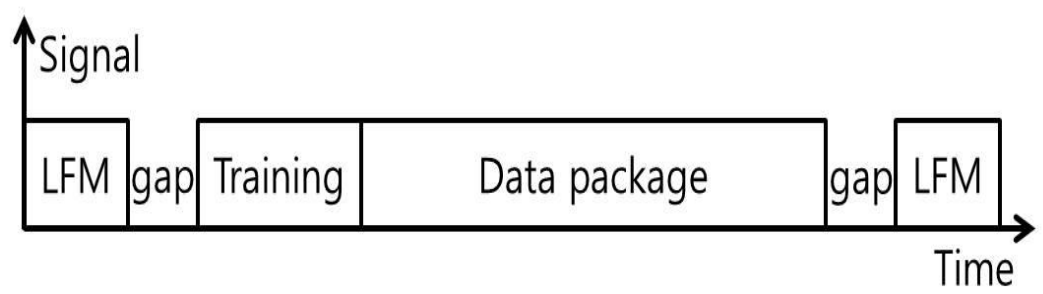

Fig.4 Packet structure

In Fig. 4, as can be seen, each packet is preceded by a linear frequency modulation (LFM) signal. The LFM is followed by a gap, plus training symbols followed by data package. At the front of the data package, a block of training symbols is used for timing synchronization and initial channel estimation. The whole packet is ended by another LFM signal, which was separated from the data package with a gap. During transmission, gaps that were sufficiently long were introduced among packets for avoiding inter-packet interference. The timing synchronization was carried out by the training symbol, which was an M-sequence of 512 bits.

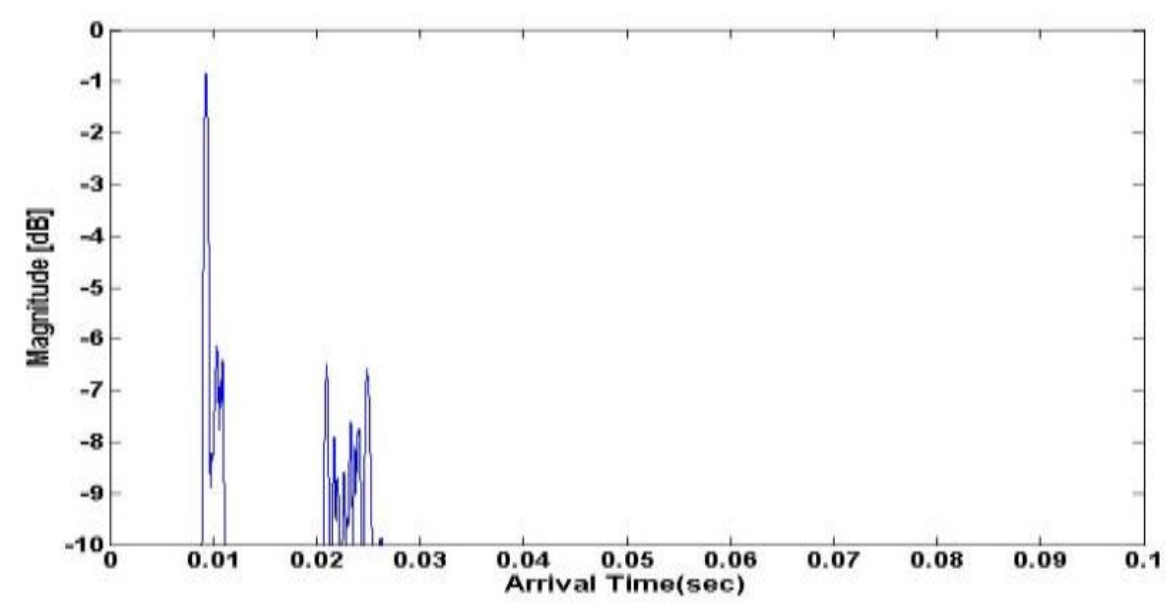

Fig.5 Underwater channel response 
In Fig 5, underwater channel response is shown during 0.1-seconds. This response was measured by using LFM signal with bandwidth of $4[\mathrm{kHz}]$. The channel gains for the secondary/third arrivals fluctuate more rapidly. This sparse channel is affected by multi-path propagation by reflection from surface and bottom. Experiment parameters are listed in Table 1. Fig. 6 shows the waveforms of transmitted and received signal, which were experiment data.

Table 1 : Parameters of UWA channel Experiment.

\begin{tabular}{lc}
\hline Source & 472 bit text \\
\hline Channel coding & $(2,1,7)$ Convolutional code \\
Input/Output bit size & $472 / 944$ bits \\
Bit rate & $1[\mathrm{kbps}]$ \\
Center frequency & $16[\mathrm{kHz}]$ \\
Sampling frequency & $192[\mathrm{kHz}]$ \\
Modulation & $\mathrm{QPSK}$ \\
Distance & $400[\mathrm{~m}]$ \\
Depth & $\mathrm{TX}:[2 \mathrm{~m}], \mathrm{RX}:[30 \mathrm{~m}]$ \\
\hline
\end{tabular}

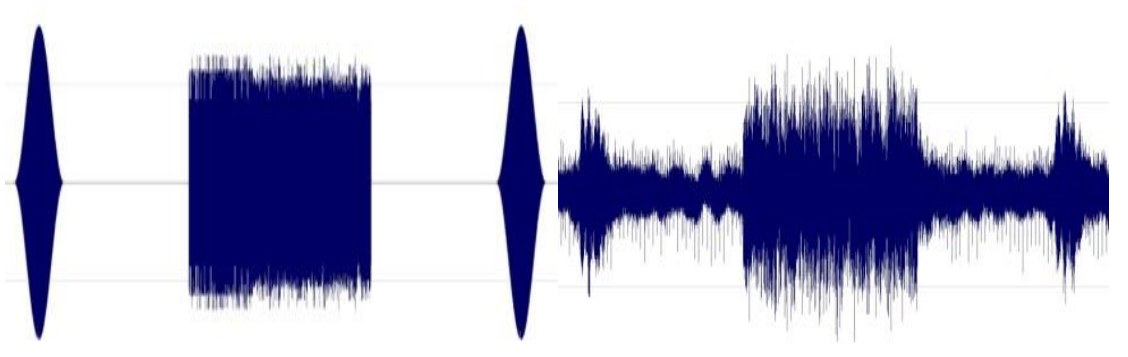

(a)

(b)

Fig.6 The waveforms of (a) transmitted signal and (b) received signal

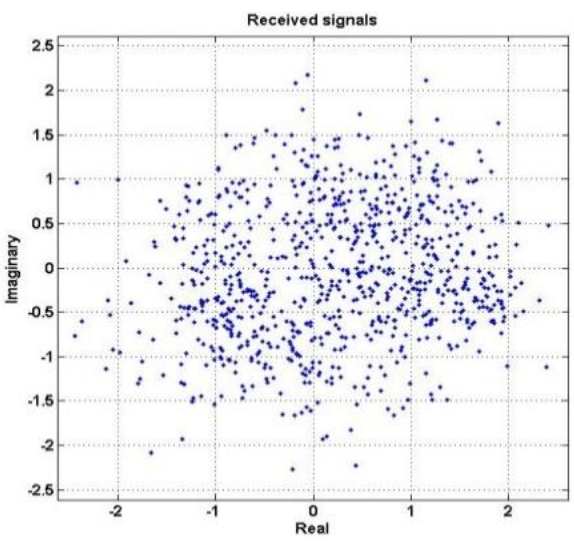

(a)

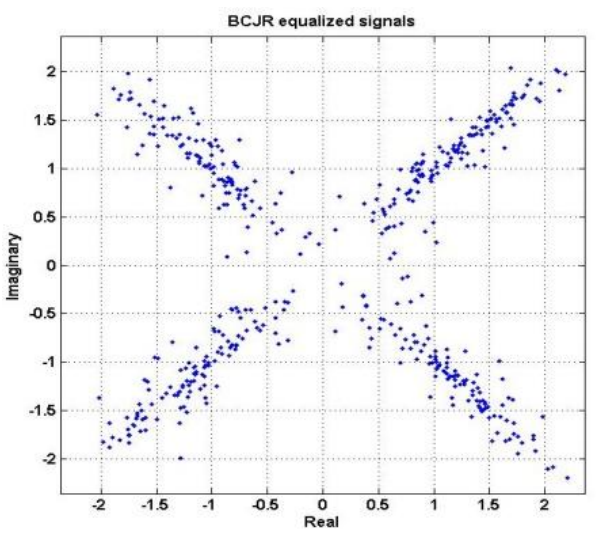

(b)

Fig.7 Scatter plot of (a) received signal and (b) BCJR equalized signal

The scatter plots as shown in Fig. 7, it's obvious that most of the symbols can be properly classified except a few. We confirmed the proposed system works effectively in UW communication. Based on the proposed model as shown in Fig.1, Fig. 8 shows number of errors of iterative BCJR equalization. We confirmed that the performance is gradually increased as iteration numbers are increased. We can achieve the performance gain as error free after third iterations. 


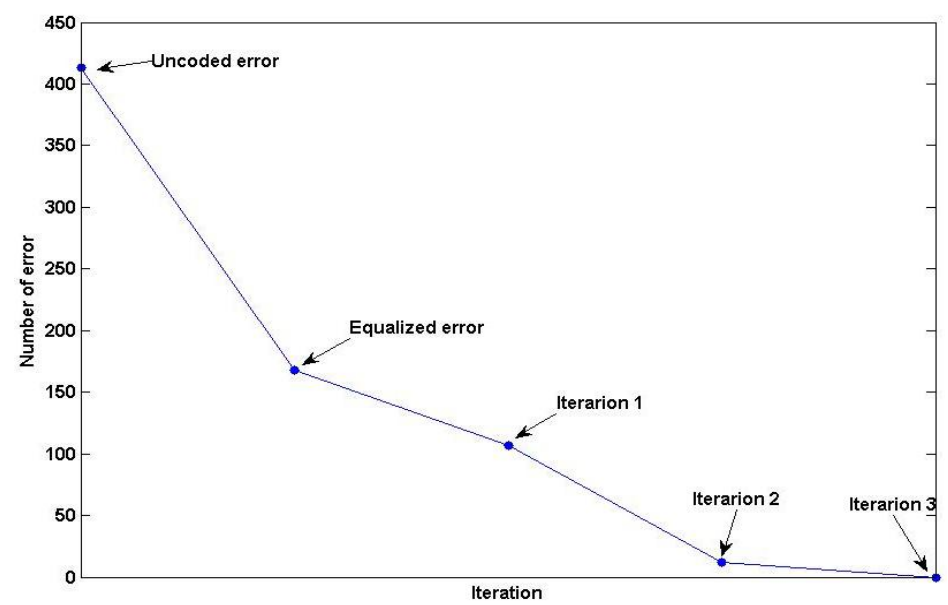

Fig.8 The number of errors for iterative BCJR equalization

\section{Conclusions}

In this paper, we employ the iterative receiver structure and present more details on the structure and algorithms which has BCJR equalization with convolutional codes. The structure with fine-tuned parameters is employed to process experimental data of fixed transmitter to fixed receiver at transmission data rate of $1 \mathrm{k} \mathrm{symbol} / \mathrm{s}$. The experiment was conducted on a lake in Munkyeong city, Korea, in March 2014. In the results, the performance is gradually improved as iteration numbers are increased. We can achieve the performance gain as error free after third iterations. The results indicate that the scheme works effectively with coded bit error rate (BER) on the order of zero. It shows that how much coding gains can be obtained by increasing iteration number.

\section{Acknowledgements}

This work was supported by Defense Acquisition Program Administration and Agency for Defense Development under the contract UD130007DD.

\section{References}

[1] M. Stojanovic, J. Catipovic, and J. Proakis: Phase coherent digital communications for underwater acoustic channels., IEEE J. Ocean. Eng., Vol. 19, no. 1, Jan. (1994), pp.100-111.

[2] Daniel B. Kilfoyle and Arthur B. Baggeroer : The state of art in underwater acoustic telemetry, IEEE J. Oceanic Eng., Vol.25, no.1, Jan (2000), pp.4-27.

[3] M. Tuchler, R. Koetter, and A. Singer: Turbo Equalization : Principles and New Results, IEEE Trans. Communications, Vol.50, no.5, May (2002), pp.754-767.

[4] R. Koetter, A. C. Singer, and M. Tuchler: Turbo equalization, IEEE Signal Process. Mag., Vol. 21, no. 1, Jan (2004), pp. 67-80.

[5] B. D. Trumpis and P. L. McAdom: Performance of convolutional codes on burst noise channels in Proc. Nat. Telecommun. Conf., Los Angeles, CA, Dec (1977), pp.36:3-1 - 36:3-14.

[6] J. Salz: Optimum Mean-Square Decision Feedbac Equalization, BSTJ, 52, No. 8 October (1973), pp. 1341-73. 4

[7] K. Berberdis, T. Rontogiannis and S. Theodoridis: Efficient block implementation of the LMS based DFE, Proceedings 13th Digital Signal Processing International Conference, Vol.1, July (1997), pp. 143-146.

[8] L. R. Bahl, J. Cocke, F. Jelinek, and J. Raviv: Optimal decoding of linear codes for minimizing symbol error rate, IEEE Trans. Inf. Theory, Vol. IT-20, No. 2, Mar. (1974), pp. 284-287. 
[9] Y. Yasuda, Y. Hirata, and A. Ogawa: Optimum soft decision for Viterbi decoding. In Proc. 5th Int. Conf. Digital Satellite Commun., Genoa, Italy, Mar. (1981), pp. 251-258.

[10]K. J. Larsen: Short convolutional codes with maximal free distance for rate 1/2, 1/3 and 1/4 IEEE Trans. Inform. Theory., Vol. COM-19, May (1973), pp. 371-372. 